Proceedings of the 26th Annual International Conference of the IEEE EMBS

San Francisco, CA, USA • September 1-5, 2004

\title{
atherosclerotic Carotid Plaque Segmentation
}

\author{
C.P. Loizou ${ }^{1}$, C.S. Pattichis ${ }^{2}$, R.S.H. Istepanian ${ }^{3}$, M. Pantziaris ${ }^{4}$, A. Nicolaides ${ }^{4}$ \\ ${ }^{1}$ Dept. of Computer Science, Intercollege, P. O. Box 51604, CY-3507 Limassol, Cyprus \\ ${ }^{2}$ Dept. of Computer Science, University of Cyprus \\ ${ }^{3}$ Mobile Information and Network Technologies Research Center (MINT@K), School of Computing and Information \\ Systems, Kingston University, Kingston-Upon-Thames, London KT1 2EE, UK \\ ${ }^{4}$ Cyprus Institute of Neurology and Genetics, Nicosia, Cyprus
}

\begin{abstract}
Atherosclerosis is the major cause of heart attack and stroke in the western world. In this paper we present a computerized method for segmenting the athrerosclerotic carotid plaque from ultrasound images. The method uses the blood flow image first to detect the initial contour of the plaque, and then despeckle filtering and snakes to deform the initial contour for best fit of plaque boundaries. The accuracy and reproducibility of this method was tested using 35 longitudinal ultrasound images of carotid arteries and the results were compared with the manual delineations of an expert. The comparison showed that the computerized method gives satisfactory results with no manual correction needed in most of the cases. The true positive fraction, $\mathrm{TPF}$, true negative fraction, TNF, false negative fraction, FNF and false positive fraction, FPF, were $86.44 \%, 84.03 \%, 8.5 \%$, and $7 \%$ respectively.
\end{abstract}

Keywords - Carotid plaque, segmentation, snakes

\section{INTRODUCTION}

Carotid atherosclerosis is the primary cause of stroke and the third leading cause of death in the United States. Almost twice as many people die from cardiovascular diseases than from all forms of cancer combined. Atherosclerosis is a disease of the large and medium size arteries, and it is characterized by plaque formation due to progressive intimal accumulation of lipid, protein, and cholesterol esters in the blood vessel wall [1], which reduces blood flow significantly. Atherosclerotic plaques may develop at different sites of the vasculature, including the coronary arteries, the superficial femoral artery, the infarenal aorta and the carotid arteries at the area of the common carotid bifurcation. Atherosclerotic plaque formation initially causes compensatory enlargement of the blood vessel wall with little or no compression of the lumen [2] Traditionally the degree of artery stenosis, or narrowing, has been targeted as the marker for assessment of risk for plaque vulnerability depended on the type of plaque, and considered to cause either a complete arterial occlusion or ischemic event in the brain. The risk of stroke increases with the severity of carotid stenosis and is reduced after carotid endarterectomy [3]. The degree of internal carotid stenosis is the only well established measurement that is used to assess the risk of stroke [4]] Indeed, it is the only criterion at present used to decide whether carotid endarterectomy is indicated or not [5].
The development and testing of new methods for noninvasive assessment of plaque size and vulnerability (plaque with a high risk to rupture) will help the clinical assessment of the subject. Furthermore, non-invasive assessment of plaque characteristics will help the way in which athrerosclerotic disease is diagnosed, monitored and treated.

Some researchers have attempted to segment the carotid plaque from vascular magnetic resonance images (MRI), by using active contours to detect the artery lumen and plaque localization [6], and dynamic programming to detect the optimal borders in each MRI frame [7] Others have used a graph-searching approach to detect the wall and plaque in intravascular ultrasound images [8] There is currently no method reported for estimating plaque borders in ultrasound longitudinal images of the carotid artery.

In this paper, we present a system for the delineation of the arterial plaque from ultrasound images of the carotid artery. The system is based on snake segmentation (Section II) using a modification of the greedy algorithm and is applied as described in Section III. The results of this analysis are evaluated in Section IV and our conclusions are presented in Section V.

\section{SNAKES}

A snake contour may be represented parametrically by $v(s)=[x(s), y(s)]$, where $(x, y) \in \mathfrak{R}^{2}$ denotes the spatial coordinates of an image, and $s \in[0,1]$ represents the parametric domain. The snake adapts itself by a dynamic process that minimizes an energy function defined as follows 9]

$E_{\text {snake }}(v(s))=E_{\text {int }}(v(s))+E_{\text {image }}(v(s))+E_{\text {external }}(v(s))=$
$\int_{s}\left(\alpha(s) E_{\text {cont }}+\beta(s) E_{\text {curv }}+\gamma(s) E_{\text {image }}+E_{\text {external }}\right) d s$.

At each iteration step, the energy function is evaluated for the current point in $v(s)$, and for the points in an $m x n$ neighborhood along the arc length $s$ of the contour. Subsequently the point on $v(s)$ is moved to the new position in the neighborhood that gives the minimum energy. The term $E_{\text {int }}(v)$ in (1) denotes the internal energy derived from the physical characteristics of the snake and is given by the continuity $E_{\text {cont }}(v)$ and the curvature term $E_{\text {curv }}(v)$. This term controls the natural behavior of the 
snake. The internal energy contains a first-order derivative controlled by $\alpha(s)$, which discourages stretching and makes the model behave like an elastic string by introducing tension and a second order term controlled by $\beta(s)$, which discourages bending and makes the model behave like a rigid rod by producing stiffness. The weighting parameters $\alpha(s)$ and $\beta(s)$ can be used to control the strength of the model's tension and stiffness, respectively. Altering the parameters $\alpha, \beta$ and $\gamma$ affect the convergence of the snake. The second term in (1) $E_{\text {image }}$, represents the image energy due to some relevant features such as the gradient of edges, lines, regions and texture [9] It attracts the snake to low-level features such as brightness and edge data. Finally the term $E_{\text {external }}$ is the external energy of the snake, which is defined by the user and is optional. In our study we used a modification of the greedy algorithm as presented in 9$]$.

\section{Methodology}

\section{A. Recording of Ultrasound Images}

A total of 35 B-mode and blood flow (PW Doppler) longitudinal ultrasound images of the common carotid artery (CCA) were selected at random representing different types of atherosclerotic plaque formation with irregular geometry typically found in this blood vessel. The images were captured using the ATL HDI-5000 ultrasound scanner [10] and were recorded digitally on a magneto optical drive, with a resolution of $768 \times 756$ pixels with 256 gray levels. The image resolution was 16.66 pixels $/ \mathrm{mm}$.

The ATL HDI-5000 ultrasound scanner is equipped with an 256-element fine pitch high-resolution $50 \mathrm{~mm}$ linear array, a multi element ultrasound scan head with an extended operating frequency range of $5-12 \mathrm{MHz}$ and real spatial compound imaging. The scanner increases the image clarity using SonoCT imaging by enhancing the resolution and borders, so that interface margins can be displayed better.

Traditionally, suspected plaque formation is confirmed using color blood flow imaging. The PW Doppler used can detect blood flow at a specific depth by selecting the time interval between the transmitted and received pulses. In this work we used the blood flow image obtained for an initial snake contour estimation. The limitations of this approach i.e. using the blood flow image to locate the blood borders are the following: a) the blood sometimes hides areas of the tissue (verbarations), and $b$ ) the colour does not always fill up the places where blood has a low speed.

Plaques may be classified into the following types: type I: uniformly echolucent (black), where bright areas occupy less than $15 \%$ of the plaque area, type II: mainly echolucent, where bright echoes occupy $15-50 \%$ of the plaque area, type III: mainly echolucent, where bright echoes occupy $50-85 \%$ of the plaque area, type IV: uniformly echogenic, where bright echoes occupy more than $85 \%$ of the plaque area, type V: calcified cup with acoustic shadow so that the rest of the plaque cannot be visualized [5], [11] In this study the plaques delineated were of type II, III and IV because it is easier to make a manual delineation since the fibrous cup, which is the border between blood and plaque, is more easily identified. If the plaque is of type I, borders are not visible well. Plaques of type $\mathrm{V}$ produce acoustic shadowing and the plaque is also not visible well. Plaques of type I and $\mathrm{V}$ were therefore not delineated in our study.

\section{B. Image Normalization}

The images were normalized manually by adjusting the image linearly so that the median gray level value of blood was 15-20, and the median gray level of adventitia (artery wall) was 180-200 [11]. The scale of the gray level of the images ranged form 0 to 255 . This normalization (i.e. using blood and adventitia as reference points) was necessary in order to extract comparable measurements in case of processing images obtained by different operators or different equipment [5], [11].

\section{Manual Delineation of Plaque}

One vascular expert delineated the plaques on all $35 \mathrm{~B}-$ mode ultrasound images of the carotid artery after image normalization. The expert defined the outline of the plaque by marking 20 to 40 consecutive points on the plaque border on the B-mode image. In order to delineate the plaque on the B-mode image the expert was guided by the blood flow image. The plaque outline was drawn on the far wall (posterior) of the CCA because there, the intima media complex and the neighboring tissues are more visible. Delineations taken from the near wall are less accurate, because of overlap of echo pulses, and therefore less reproducible than those taken from the far wall [5], [11].

\section{Despeckling}

Speckle is a form of multiplicative noise, which corrupts medical ultrasound imaging making visual observation difficult. In a recent study [12] we have shown that despeckle filtering improves the expert's optical perception. Many researchers refer to speckle as the major difficulty in analyzing and segmenting ultrasound images [12][14] In this study, the linear filter $l s m v$ was used, which may be described by a weighted average calculation using sub region statistics to estimate statistical measures over $7 \times 7$ pixel windows [12]. The filter is based on first order statistics and was applied for five iterations on each image. It assumes that the speckle noise model has the following multiplicative form [12] $g_{i, j}=f_{i, j} n_{i, j}$ with $i, j \in N$, where $g_{i, j}$ represents the noise pixel in the middle of the moving window, $f_{i, j}$ represents the noise-free pixel and $n_{i, j}$ is a Rayleigh distributed noise on pixel. The $l s m v$ algorithm is based on the following equation [12]

$f_{i, j}=\bar{g}_{i, j}+k_{i, j}\left(g_{i, j}-\bar{g}_{i, j}\right)$ 
where $f_{i, j}$ is the new estimated pixel, $g_{i, j}$ is the old pixel in the middle of a moving window, $\bar{g}_{i, j}$ is the local mean value of an $N_{1} x N_{2}$ region, $k_{i, j}$ is a weighting factor with $k \in[0 . .1]$, and $i, j$ are the absolute pixel coordinates. The factor $k_{i, j}$ is a function of the local statistics in a moving window and is derived as $k_{i, j}=\sigma^{2} /\left(\bar{g}_{i, j}^{2} \sigma^{2}+\sigma_{n}^{2}\right)$, where $\sigma^{2}$ and $\sigma_{n}^{2}$ are the variance in the moving window and the variance of noise in the image, respectively [12]. The variance of noise for the logarithmic compressed image is $\sigma_{n}=g_{i, j} / \sigma_{i, j}^{2} 13$

\section{E. Snake Contour Initialization}

It is importnat to position the initial snake contour as close as possible to the area of interest otherwise the snake may be trapped into local minima or false edges, and converge in a wrong location. We have therefore developed an initialization procedure where the outline of the blood flow is used to detect the initial contour placement. The initialisation procedure may be described as follows (see also Fig. 1 at the Results Section): a) Cross correlate the Bmode image (see Fig. 1a) with the blood flow image (see Fig. 1b) and extract the blood flow area. b) Dilate the extracted blood flow area to close small gaps and remove small regions. c) From the dilated blood flow image, detect the blood flow edge contour (see Fig. 1c). d) Mark a region of interest on the edge contour (task carried out by the expert, rectangle shown in Fig. 1c), where the lower or upper boundary of plaque is covered to use it as initial snake contour. e) Sample the initial snake contour at 20 to 40 consecutive snake points to construct an interpolating Bspline (see Fig. 1d). f) Connect the first and the last snake points on the initial contour to form a close contour. g) Despeckle the B-mode image by the $l s m v$ filter. h) Map the initial plaque contour on the B-mode image. i) Deform the initial contour by a snake to accurately locate the plaqueblood borders, and j) Save the final plaque contour and display it on the B-mode image (see Fig. 1f).

\section{F. Evaluation of the Segmentation Method}

To evaluate the performance of the proposed segmentation method, we compared the manually identified borders with the computerized detected borders defined by an expert. The intra- and inter-observer variability caused by multiple experts, was not taken into account in this study. Let $G T$ denote the segmented area representing ground truth, $\overline{G T}$ its complement, and $A S$ the segmented area obtained by the computerized approach. The receiver operating characteristics (ROC) analysis was used to assess the specificity and sensitivity of the method by the truepositive fraction (TPF) and false-positive fraction (FPF) detected [15] respectively. The $T P F$, is calculated when the expert detects a plaque (when plaque is present) and the computerized method identifies it as so, whereas the FPF, is calculated when the expert detects no plaque and the computerized method incorrectly detects that there is plaque present. The $T N F$ fraction is calculated when the expert identifies no plaque and the computerized method identifies it as so (absent), whereas the $F N F$ is calculated when the expert identifies plaque presence and the computerized method incorrectly identifies plaque absence. Ratios of overlapping areas, can also be assessed by applying the similarity kappa index ( $K I$ ) [16] and the overlap index [17]. These indices were computed as follows

$$
\begin{array}{ll}
T P F=\frac{A S \cap G T}{G T}, & F P F=\frac{|A S-G T|}{\overline{G T}}, \\
K I=2 \frac{G T \cap A S}{G T+A S}, & \text { overlap }=\frac{G T \cap A S}{G T \bigcup A S}
\end{array}
$$

where $\bigcap$ denotes the intersection and $U$ the union of the two areas. The intersection gives the probability that both $A S$ and $G T$ occur and the union is the probability that either $A S$ or $G T$ occur.

\section{RESULTS}

Figure 1 illustrates the original ultrasound image of the carotid artery with a plaque at the far wall (a), the blood flow image (b), the initial blood flow edge contour and the user selected initial contour (enclosed in the rectangle) (c), the user selected sampled initial snake contour superimposed on the original image (d), the manual segmentation results of the expert (e), and the segmentation results of the proposed snakes method (f). As shown the manual and the computerized segmentation results (e) vs (f) are optically very similar suggesting that the two segmentation methods can be interchangeable.

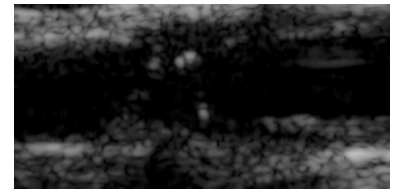

a) Original Image

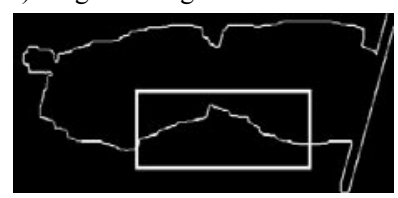

c) Initial Blood flow edge contour

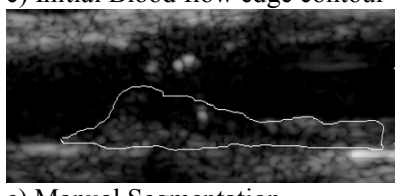

e) Manual Segmentation

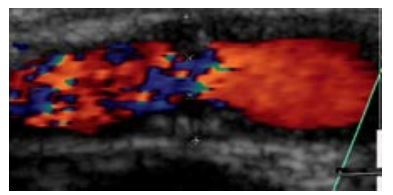

b) Blood Flow Image

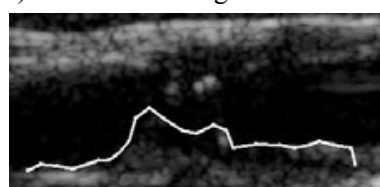

d) Sampled initial snake contour

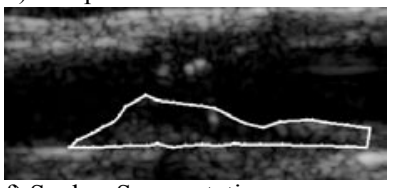

f) Snakes Segmentation
Fig. 1. a) Original ultrasound image of a carotid artery with plaque, b) blood flow image, c) initial blood flow edge contour with expert selected area for the initial contour, d) sampled initial snake contour, e) manual segmentation of plaque, and f) Snakes segmentation of plaque. 
Table I shows a comparison of the plaque segmentation scores by an expert and the proposed segmentation method. It is shown that the proposed method agrees with the expert in $84.03 \%$ of the cases (TNF) by correctly detecting no plaque, in $86.44 \%$ of the cases $(T P F)$ by correctly detecting a plaque, and disagrees with the expert, in $7 \%$ of the cases ( $F P F)$ by detecting plaque, and in $8.5 \%$ ( $F N F)$ of the cases by detecting no plaque.

Ideally, the computerized method should have a $100 \%$ score for $T P F$ and $T N F$ and a $0 \%$ score for FPF and $F N F$ for the images analyzed. The similarity kappa index, $\mathrm{KI}$, and the overlap index between the manual method and the proposed segmentation method, were $85.5 \%$ and $74 \%$ respectively, which are considered very satisfactory.

TABLE I

COMPARISON OF Plaque DETECTION By AN EXPERT AND By The Proposed SNAKES METHOd ON 35 IMAGES

\begin{tabular}{l||c|c}
\hline System detects & Expert detects no plaque & Expert detects plaque \\
\hline No plaque & $\mathrm{TNF}=84.03 \%$ & $\mathrm{FNF}=8.5 \%$ \\
\hline Plaque & $\mathrm{FPF}=7 \%$ & $\mathrm{TPF}=86.44 \%$ \\
\hline
\end{tabular}

\section{CONCLUDING REMARKS}

In this study we have proposed and evaluated a new computer based method that can be used for the segmentation of atherosclerotic carotid plaque. The initial estimate of the contour of the plaque is carried out on the PW Doppler blood flow image and subsequently mapped on the B-mode image. This initial contour is then deformed using snakes to find the final contour. The results of this study show that the proposed segmentation method is very satisfactory without requiring manual correction in most of the cases. The true positive fraction, TPF, true negative fraction, TNF, false negative fraction, FNF and false positive fraction, FPF, were $86.44 \%, 84.03 \%, 8.5 \%$, and $7 \%$ respectively. Therefore, the proposed method gives results comparable to the manual delineation procedure.

The method presented is to the best of our knowledge the first computerized approach for plaque segmentation from ultrasound images of the carotid artery. Such a computerized method cannot only reduce significantly the time required for the image analysis, but also it can reduce the subjectivity that accompanies manual measurements. The method will be further evaluated on a larger number of ultrasound images and on multiple experts' evaluation. Furthermore, it is expected that the segmentation method will be incorporated into an integrated system enabling the texture analysis of the segmented plaque (as documented in [18], providing an automated system for the early diagnosis and the assessment of the risk of stroke.

\section{ACKNOWLEDGMENT}

This work was partly funded through the project Integrated System for the Support of the Diagnosis for the
Risk of Stroke (IASIS), of the $5^{\text {th }}$ Annual Program for the Financing of Research of the Research Promotion Foundation of Cyprus as well as, through the project Integrated System for the Evaluation of Ultrasound Imaging of the Carotid Artery (TALOS), of the Program for Research and Technological Development 2003-2005, of the Research Promotion Foundation of Cyprus.

\section{REFERENCES}

[1] C. K. Zarins, C. Xu, S. Glagov, "Atherosclerotic enlargement of the human abdominal aorta", Elsevier Sc. Ireland, pp.157-164, 2001.

[2] S. Glagov, E. Weisenberg, C. K. Zarins, R. Stankunavicius, "Compensatory enlargement of human athrerosclerotic coronary arteries", NEJM, vol. 316, pp. 1371-1375, May 1987.

[3] ACAS Clinical advisory: carotid endarterectomy for patients with asymptomatic internal carotid artery stenosis, Stroke, vol. 25, no. 12, pp. 2523-2524, 1994.

[4] Executive Committee for the Asymptomatic Carotid Atherosclerosis Study, "Endarterectomy for asymptomatic carotid stenosis," J. Am. Med. Assoc., pp. 273:1421-8, 1995.

[5] A. Nicolaides, M. Sabetai, S. K. Kakkos, S. Dhanjil, T. Tegos, J. M. Stevens, "The Asymptomatic Carotid Stenosis and Risk of Stroke study", Int. Angiol., vol. 22, no. 3, pp. 263-272, 2003.

[6] G. J. Adams, G. W. Vick, C. M. Ballantyne, W. Insull, J. D. Morrisett, "Estimation of carotid atherosclerotic plaque volume in vivo magnetic resonance images", Proc. EMBS/BMES Conf., Houston, TX, USA, pp. 1072-1073, Oct. 23-26, 2002.

[7] F. Yang, G. A. Holzapfel, Ch. A. J. Schulze-Bauer, R. Stollberger, D. Thedens, L. Bolinger, A. Stolpen, M. Sonka, "Segmentation of wall and plaque in in-vitro vascular MR images", The Int. J. of Cardiov. Imag., vol. 19, pp. 419-428, 2003.

[8] X. Zhang, C. R. McKay, M. Sonka, "Tissue characterization in intravascular ultrasound images", IEEE Trans. Med. Imag., vol. 17, no. 6, pp. 889-899, 1998.

[9] D. Williams, M. Shah, "A fast algorithm for active contour and curvature estimation", GVCIP: Imag. Und., vol. 55, no. 1, pp. 14-26, 1992.

[10] A Philips Medical System Company, "Comparison of image clarity, SonoCT real-time compound imaging versus conventional 2D ultrasound imaging", ATL Ultrasound, Report, 2001.

[11] T. Elatrozy, A. Nicolaides, T. Tegos, A. Zarka, M. Griffin, M. Sabetai, "The effect of B-mode ultrasonic image standardization of the echodensity of symptomatic and asymptomatic carotid bifurcation plaque", Int. Angiol., vol. 17, no. 3, pp. 179-186, 1998.

[12] C. Loizou, C. Christodoulou, C. S. Pattichis, R. S. H. Istepanian, M. Pantziaris, A. Nicolaides, "Speckle reduction in ultrasound images of atherosclerotic carotid plaque", DSP 2002, IEEE $14^{\text {th }}$ Int. Conf. on Digital Signal Proc., vol. 2, pp. 525-528, 2002.

[13] V. Dutt, "Statistical analysis of ultrasound echo envelope", Ph.D. Dissertation, Mayo Graduate School, Rochester, MN, 1995.

[14] C. Loizou, C. S. Pattichis, R. S. H. Istepanian, M. Pantziaris, "Segmentation of carotid artery by using snakes," Medicon, 2004, July 31-August 5, Ischia, Naples-Italy, 2004, submitted.

[15] C. Metz, "Basic principles of ROC analysis," Semin. Nucl. Medic., vol. 8, pp. 283-298, 1978.

[16] A. Zijdenbos, B. Dawant, R. Margolin, et al., "Morphometric analysis in white matter lesions in MR images: Method and validation," IEEE Trans. Med. Imag., vol. 13, no. 4, pp. 716-724, Dec. 1994.

[17] A. Kelemen, G. Szekely, G. Gerig, "Elastic model-based segmentation of 3-D neuroradiological data sets," IEEE Trans. Med. Imag., vol. 18, no. 10, pp. 828-839, Oct. 1999.

[18] C. I. Christodoulou, C. S. Pattichis, M. Pantziaris, A. Nicolaides, "Texture based classification of atherosclerotic carotid plaques", IEEE Trans. Med. Imag., vol. 22, no. 7, pp. 902-912, July 2003. 